\title{
Operational optimization of a building-level integrated energy system considering additional potential benefits of energy storage
}

\author{
Sai Liu', Cheng Zhou ${ }^{2}$, Haomin Guo ${ }^{3}$, Qingxin Shi ${ }^{4}$, Tiancheng E. Song ${ }^{5}$, lan Schomer ${ }^{4}$ and Yu Liu ${ }^{5^{*}}$ (D)
}

\begin{abstract}
As a key component of an integrated energy system (IES), energy storage can effectively alleviate the problem of the times between energy production and consumption. Exploiting the benefits of energy storage can improve the competitiveness of multi-energy systems. This paper proposes a method for day-ahead operation optimization of a building-level integrated energy system (BIES) considering additional potential benefits of energy storage. Based on the characteristics of peak-shaving and valley-filling of energy storage, and further consideration of the changes in the system's load and real-time electricity price, a model of additional potential benefits of energy storage is developed. Aiming at the lowest total operating cost, a bi-level optimal operational model for day-ahead operation of BIES is developed. A case analysis of different dispatch strategies verifies that the addition of the proposed battery scheduling strategy improves economic operation. The results demonstrate that the model can exploit energy storage's potential, further optimize the power output of BIES and reduce the economic cost.
\end{abstract}

Keywords: Building-level integrated energy system, Energy storage, Additional potential benefits, Bi-level optimization

\section{Introduction}

According to reports issued by the International Energy Agency and the U.S. Energy Information Administration, energy consumption of global buildings accounts for approximately $32 \%$ of total consumption. Among which, the energy consumption of residential and commercial buildings account for about $20 \%$ of the total transmitted energy in the world, and the total $\mathrm{CO}_{2}$ emissions from building energy consumption account for $30 \%$ of the total [1-4]. At present, China's building energy consumption accounts for more than $27 \%$ of its total energy consumption [5]. Moreover, with continuous urbanization and adjustment of industrial structure,

\footnotetext{
* Correspondence: yuliu@seu.edu.cn

${ }^{5}$ School of Electrical Engineering, Southeast University, Nanjing 210096, Jiangsu Province, China

Full list of author information is available at the end of the article
}

building energy consumption continues to increase, which may further exacerbate the problems of environmental degradation and resource scarcity. Therefore, optimizing the scheduling strategy of the energy system in a BIES and promoting the efficient use of various types of clean energy are of great significance for energy use improvement, system operating costs reduction, and sustainable urban development [6-8].

At present, research on BIES mainly focuses on the system model, optimization strategy, and solution algorithm. Reference [9] constructs two-dimensional and three-dimensional models of distributed energy systems, which can effectively reduce the operating cost of a BIES. However, the energy consumption equipment only has a few selections with low strategic flexibility. Reference [10] proposes an economic optimization scheduling method for multi-type cooling systems in large 
buildings, which reduces the energy consumption of the cooling system. However, it lacks a comprehensive consideration of the combined cooling, heating and power systems. Taking the intelligent building group as a multi-stakeholder application scenario, reference [11] constructs a day-ahead optimization sharing model, and the Shapley value method is used to allocate the income of each building to increase the total income of the cluster. Reference [12] studies the energy distribution strategy between buildings. On the basis of the minimum electricity cost of cluster electric vehicles, the model considers the energy sharing and the lowest scheduling cost between buildings and establishes a day-ahead optimization scheduling model. From the perspective of energy utilization, references $[13,14]$ tackle the energy waste problem caused by the mismatch of the chargeside electric-heat ratio of the interconnected CCHP-type microgrid sources by energy sharing, so the economic competitiveness of microgrid operation is improved. Aiming at air-conditioning load, the outdoor temperature and real-time electricity price are transformed into a fuzzy set in [15]. This is solved with the lowest electricity cost and minimum room temperature violation as the objective functions can reduce the impact of forecast uncertainty on BIES operating costs. Reference [16] investigates the influence of the electricity tariff on energy flexibility in buildings and associated energy costs, including real-time pricing, two-level daynight tariff and critical-peak pricing with both fixed and variable feed-in price components. The flexibility of user heating demand is considered in [17] and a flexible demand response model of electric-heat load is established. This effectively reduces the peak-to-valley difference of the load and the total operating cost of a BIES. In [18], artificial neural networks are integrated into an optimization problem for a price-based demand response to solve various electricity prices and building thermal conditions, while reference [19] calculates the flexibility potential by evaluating the capability of buildings to dynamically change their demand profiles according to external penalty signals, such as energy price, carbon emission, etc.

Energy storage equipment plays an important role in cutting peaks and filling valleys, smoothing fluctuations in renewable energy, and alleviating the imbalance between energy production and consumption. It can improve the economic efficiency and flexibility of a BIES. The application of a combined heat and power system and energy storage in an IES is analyzed in [20], and the economic benefits to system operation of battery energy storage are studied by solving the economic optimization model. The results show that combination of heat and power system and energy storage can reduce the operating cost of the microgrid. Reference [21] proposes a mixed-integer linear programming method to solve the problem of microgrid economic dispatch. The study linearizes the models of traditional micro power and energy storage, and compares with traditional genetic algorithms in terms of speed of solution and accuracy. Based on an improved gravitational search algorithm, reference [22] solves the problem on the premise of energy storage and new energy generation, and optimizes the output of dispatchable micro power sources such as fuel cells and diesel engines in the microgrid. Power storage equipment is introduced in [23] as a key tool for demand response in household energy management while the proposed model maximizes the benefits to consumers and network operators with reduced electricity costs and investment costs. Using a variety of energy storage modes including thermal inertia and the flexibility of cooling and heating loads, a coordinated scheduling model is established in [24] for multi-energy complementary systems to determine the optimal control strategy. Most studies focus on an optimal scheduling and solution algorithm of an IES with energy storage, while considering the effect of energy storage and renewable energy on peak shaving and valley filling. However, the operational status of energy storage is relatively fixed because of the limitation of distributed energy output and IES load demand. Those studies do not consider the influence of the daily electricity price fluctuation and the pattern of load change on the optimization of IES operation. As a result, the full picture of the dispatching role of energy storage is not shown.

This paper takes the user-side building as the research object and integrates the technology of combined cooling, heating and power into a BIES. Energy storage for enhancing the coupling of equipment is introduced, and the flexibility and economic efficiency are further improved. Firstly, an operating characteristic model of the distributed energy equipment is established considering the factors in the energy consumption of the BIES. Secondly, an energy storage model is established that completely considers the impacts of load deficiencies, realtime electricity prices, and the additional potential benefits of energy storage. Thirdly, a bi-level optimal operation model for day-ahead operation with the goal of the lowest total operating cost is established. Finally, a practical testing cases are analyzed to further optimize the scheduling strategy of energy storage based on total operating cost reduction and peak load shifting to exploit its potential.

\section{Modeling of BIES}

\subsection{Typical structure}

A BIES has the distinct characteristics of residential users, and can provide various forms of energy according to users to achieve the optimal use of energy resources 
by combining existing energy, resources, technical conditions etc. Compared with other energy distribution systems, a BIES has higher operational stability, energy efficiency and economic benefits. The structure diagram of a BIES is shown in Fig. 1, which shows a photovoltaic unit (PV), wind turbine (WT), gas turbine (GT), gas boiler (GB), electric chiller (EC), absorption chiller (AC), Electric energy storage (ES) and a heat recovery boiler (HRB).

\subsection{Modeling of equipment operating characteristic}

\subsubsection{Gas turbine}

Gas turbines are the core equipment of a combined cooling, heating and power system, which can be expressed as:

$$
\begin{aligned}
& P_{\mathrm{GT} . \mathrm{i}}^{\mathrm{e}}(t)=P_{\mathrm{GT} . \mathrm{i}}^{\mathrm{g}}(t) H_{\mathrm{gas}} \eta_{\mathrm{GT}}^{\mathrm{e}} \\
& P_{\mathrm{GT} . \mathrm{i}}^{\mathrm{h}}(t)=P_{\mathrm{GT} . \mathrm{i}}^{\mathrm{g}}(t) H_{\mathrm{gas}}\left(1-\eta_{\mathrm{GT}}^{\mathrm{e}}-\eta_{\mathrm{GT}}^{\text {loss }}\right)
\end{aligned}
$$

where $P_{\mathrm{GT} . \mathrm{i}}^{\mathrm{e}}(t), P_{\mathrm{GT} . \mathrm{i}}^{\mathrm{h}}(t)$ and $P_{\mathrm{GT} . \mathrm{i}}^{\mathrm{g}}(t)$ are the output electric power, output thermal power, and natural gas consumption of gas turbine $i$ at time $t$, respectively. $\eta_{\mathrm{GT}}^{\mathrm{e}}$ is the net power generation efficiency of the gas turbine, and $\eta_{\mathrm{GT}}^{\text {loss }}$ is the gas turbine energy loss rate. $H_{\text {gas }}$ is the low calorific value of natural gas and this paper takes $H_{\text {gas }}$ as $9.78 \mathrm{kWh} / \mathrm{m}^{3}$.

\subsubsection{Gas boiler}

As a backup heat source in the BIES, gas boilers play a role in peak shaving and can be expressed as:

$$
P_{\mathrm{GB} . i}^{\mathrm{h}}(t)=P_{\mathrm{GB} . i}^{\mathrm{g}}(t) H_{\mathrm{gas}} \eta_{\mathrm{GB}}^{\mathrm{h}}
$$

where $P_{\mathrm{GB} . i}^{\mathrm{h}}(t)$ and $P_{\mathrm{GB} . i}^{\mathrm{g}}(t)$ are the output thermal power and consumed natural gas of gas boiler $i$ at time $t$, respectively. $\eta_{\mathrm{GB}}^{\mathrm{h}}$ is the heating efficiency of the gas boilers.

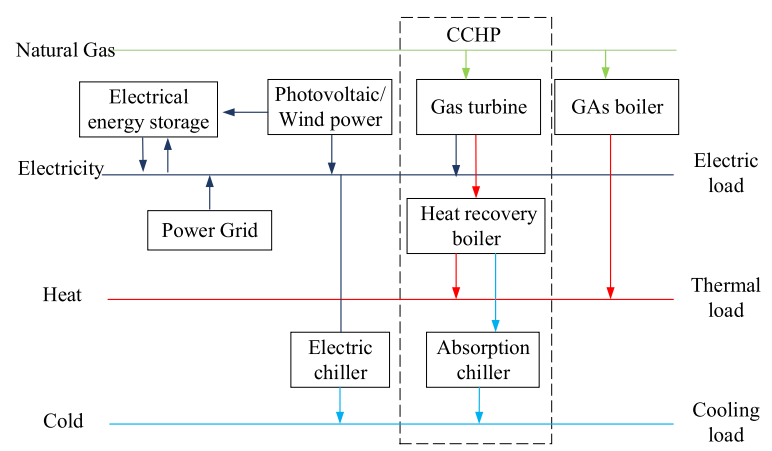

Fig. 1 Building-level integrated energy system structure

\subsubsection{Absorption chiller}

Absorption chillers use the characteristics of refrigerant vaporization to absorb heat to achieve the purpose of external cooling, and can be expressed as:

$$
P_{\mathrm{AC}}^{\mathrm{c}}(t)=C O P_{\mathrm{AC}} P_{\mathrm{AC}}^{\mathrm{h}}(t)
$$

where $P_{\mathrm{AC}}^{\mathrm{c}}(t)$ and $P_{\mathrm{AC}}^{\mathrm{h}}(t)$ are the output cold power and consumed residual calories of absorption chiller $i$ at time $t$, respectively. $C O P_{\mathrm{AC}}$ is the refrigeration energy efficiency ratio of the absorption chillers.

\subsubsection{Electric chiller}

Refrigerators are compressor-type loads, which can provide additional cooling when the refrigeration device of an IES or cold storage and cooling are insufficient. They play the role of peak shaving and can be expressed as:

$$
P_{\mathrm{EC}}^{\mathrm{c}}(t)=C O P_{\mathrm{EC}} P_{\mathrm{EC}}^{\mathrm{e}}(t)
$$

where $P_{\mathrm{EC}}^{\mathrm{c}}(t)$ and $P_{\mathrm{EC}}^{\mathrm{e}}(t)$ are the output cold power and consumed electric power of the electric refrigerators, respectively. $C O P_{\mathrm{EC}}$ is the refrigeration energy efficiency ratio of the electric refrigerators.

\subsubsection{Electrical energy storage}

Battery devices can smooth the uncertainty of renewable energy output. They can be used to improve the optimal allocation capability of IES in a large time and space. To increase the capacity of renewable energy consumption. Battery devices can be expressed as:

$$
S O C_{\mathrm{ES}}(t)=\frac{S_{\mathrm{ES}}(t)}{S_{\mathrm{ES} . \text { rate }}}
$$

where $S O C_{\mathrm{ES}}(t)$ and $S_{\mathrm{ES}}(t)$ are the state of charge and remaining capacity of the electrical energy storage, respectively. $S_{\mathrm{ES}}$. rate is the rated capacity of the electrical energy storage.

\section{Additional potential benefits of energy storage}

In a BIES, the development and addition of electricity storage technology help to make full use of clean energy that is difficult to connect to the grid, reduce the curtailment of wind and solar power, and minimize the output fluctuation of gas turbines. Through reasonable and effective scheduling and coordinated optimization among various energy subsystems, the efficiency of energy storage can be maximized and the invisible economic scheduling role of the battery can be brought into operation.

In current research on the optimal operation of a BIES, the battery mainly cooperates with distributed energy sources to supply the loads, and plays the role of peak-shaving and valley-filling. Most research is focused on reducing the amount of wind and solar energy curtailment, optimizing energy storage configuration, 
capacity, etc. The operating state of the battery is fixed because of the limitation of distributed energy output and BIES load demand. The charging/discharging scheduling method of the battery fails to adjust the operating state according to changes in electricity prices. This is not conducive to maximizing the dispatching role of the battery. In order to fully use the battery in promoting economic dispatch in energy interactive benefits, and to improve the overall economic benefits of the system, this paper determines the battery charging and discharging schedule from a dynamic perspective by fully considering the fluctuation of the electricity price, the change pattern of the load and the change in interactive power.

The additional potential income of energy storage refers to the income brought by the current state of charge and discharge when the battery participates in the "energy transfer" process of a BIES. In essence, on a basis of not changing the basic function of energy storage, the battery is charged at a low price in order to be sold at a higher price at a certain time in the future, thereby adding part of the revenue to the BIES.

To pursue greater economic benefits of a BIES, it is necessary to make full use of the difference in electricity prices at different times and fully mobilize the dispatchability of the battery. To fully consider the impact of the generation shortage and real-time electricity price, this paper establishes the objective function of the additional potential income of energy storage as follows:

$$
\begin{aligned}
& P_{N G S}(t)=P_{\mathrm{Load}}^{\mathrm{e}}(t)-\sum_{i} P_{i}(t) \\
& \sum_{i} P_{i}(t)=\sum_{i} P_{\mathrm{GT} . i}^{\mathrm{e}}(t)+P_{\mathrm{PV}}(t)+P_{\mathrm{WT}}(t) \\
& P_{\mathrm{Load}}^{\mathrm{e}}(t)=P_{\text {load }}^{\mathrm{e}}(t)+P_{\mathrm{EC}}^{\mathrm{e}}(t)
\end{aligned}
$$

where $P_{N G S}(t)$ is the net generation power shortage, $P_{i}(t)$ is the output power of each energy device, $P_{\mathrm{Load}}^{\mathrm{e}}(t)$ is the electricity demand from users, and $P_{\text {load }}^{\mathrm{e}}(t)$ is the electrical load at time $t . P_{\mathrm{PV}}(t)$ and $P_{\mathrm{WT}}(t)$ are the photovoltaic and wind turbine outputs, respectively.

To ensure that the battery will meet the conditions of discharging during a large generation shortage and charging during a small generation shortage, the following coefficients $\alpha_{\mathrm{NGS}}(t)$ and $\beta_{\mathrm{NGS}}(t)$ are set as:

$$
\begin{aligned}
& \alpha_{\mathrm{NGS}}(t)=k_{\mathrm{dis}} \frac{P_{\mathrm{NGS}}(t)}{P_{\mathrm{NGS}_{-} \max }} \\
& \beta_{\mathrm{NGS}}(t)=k_{c h} \frac{P_{\mathrm{NGS}_{-} \max }-P_{\mathrm{NGS}}(t)}{P_{\mathrm{NGS}} \text { max }}
\end{aligned}
$$

where $\alpha_{\mathrm{NGS}}(t)$ and $\beta_{\mathrm{NGS}}(t)$ are the battery discharge and charge coefficients, respectively. $P_{\mathrm{NGS} \_ \text {max }}$ is the maximum net load shortfall power, while $k_{c h}$ and $k_{\text {dis }}$ represent the proportionality of the charge and discharge coefficient, respectively. When the shortage of power at time $t$ is large, $\alpha_{\mathrm{NGS}}(t)$ is high and the battery is more likely to discharge. In contrast, when the shortage of power at time $t$ is small, $\beta_{\mathrm{NGS}}(t)$ is large and the battery is more likely to be charged. Thus, $\alpha_{\mathrm{NGS}}(t)$ and $\beta_{\mathrm{NGS}}(t)$ can effectively identify the generation shortage and instruct battery charging and discharging.

The additional potential revenue function of energy storage is given as:

$$
\begin{aligned}
F_{\mathrm{ES}} & =F_{\mathrm{ES} \_ \text {dis }}+F_{\mathrm{ES} \_c h} \\
F_{\mathrm{ES} \_ \text {dis }} & =\sum_{t=1}^{T} \mathrm{~A}_{\mathrm{NGS}} P_{\mathrm{ES}}^{\mathrm{dis}}(t) \\
& =\sum_{t=1}^{T}\left(\alpha_{\mathrm{NGS}}(t)+\delta_{\mathrm{dis}}\left(\rho_{\mathrm{se}}(t)-\rho_{\mathrm{se}_{-} \max }\right)\right) P_{\mathrm{ES}}^{\mathrm{dis}}(t)
\end{aligned}
$$

$$
\begin{aligned}
& F_{\mathrm{ES} \_c h}=\sum_{t=1}^{T} \mathrm{~B}_{\mathrm{NGS}} P_{\mathrm{ES}}^{\mathrm{ch}}(t) \eta_{\mathrm{ES}}^{\mathrm{ch}} \eta_{\mathrm{ES}}^{\mathrm{dis}} \\
& =\sum_{t=1}^{T}\left(\beta_{\mathrm{NGS}}(t)+\delta_{\mathrm{ch}}\left(\rho_{\mathrm{se} \_ \text {max }}-\rho_{\mathrm{be}}(t)\right) P_{\mathrm{ES}}^{\mathrm{ch}}(t) \eta_{\mathrm{ES}}^{\mathrm{ch}} \eta_{\mathrm{ES}}^{\mathrm{dis}}\right.
\end{aligned}
$$

where $F_{\mathrm{ES}}$ is the additional potential economic benefit of energy storage. $F_{\mathrm{ES} \_ \text {dis }}$ and $F_{\mathrm{ES} \_ \text {ch }}$ are the battery discharge and charge benefits, respectively. $\rho_{\mathrm{se}}(t)$ is the price of electricity sold by BIES at time $t, \rho_{\text {se }}$ max is the maximum price of electricity sold on a day, and $\rho_{\text {be }}(t)$ is the price of electricity purchased by the BIES from the distribution network at time $t . \delta_{\mathrm{ch}}$ and $\delta_{\text {dis }}$ are the respective charge and discharge coefficients following the changes in real-time electricity prices. $A_{\mathrm{NGS}}$ is the correlation coefficient between battery discharge status and real-time electricity price and generation shortage, while $\mathrm{B}_{\mathrm{NGS}}$ is the correlation coefficient between the SOC of the battery and the real-time electricity price and generation shortage. $P_{\mathrm{ES}}^{\mathrm{ch}}(t)$ and $P_{\mathrm{ES}}^{\text {dis }}(t)$ are the charging and discharging power of the battery at time $t$, respectively. The discharge of the battery under the current higher electricity price will gain more benefit than the discharge at a lower electricity price. If the power shortage is also large at the time, the battery discharge benefit will increase further. In the same way, when the battery is charged under the current low electricity price and discharged when the electricity price is high in the future, additional benefit will be obtained. Similarly, if the power shortage is also small at this time, the battery charging benefit will be further increased. To achieve the scheduling strategy of battery charging at low electricity price and discharging at high electricity price, the 
revenue should be increased as much as possible when optimizing the function $F_{\mathrm{ES}}$, so as to ensure the optimal charge/discharge scheduling strategy of the battery.

\section{Economical optimal operational model considering additional potential benefits of energy storage}

Considering the cost and environmental benefits of each equipment, the curtailment of wind and solar power should be avoided, and the use of clean energy should be treated with higher priority. The gas turbine adopting the 'cogeneration' method uses a waste heat recovery system to recover heat energy. When heat supply is insufficient, a gas boiler or heat storage tank is used to compensate for the heat generation shortage. In this paper, a bi-level optimization model of economic dispatch considering the additional potential benefits of electricity storage is established for the economic optimization of a BIES. The upper-level model targets the total cost of the BIES, including operating costs, interaction costs with the distribution network, and natural gas costs. The lower-level model targets the additional potential benefits of electricity storage. The transfer relationship of decision variables between the upper level and the lower level is shown in Fig. 2.

Firstly, the upper level transmits the dispatching operation status of distributed energy equipment to the lower level, while the lower level returns the optimized battery charge and discharge state to the upper level to continue the optimization. The upper level then revises the total economic cost based on the optimization results of the lower level, optimizes the dispatch operation of distributed energy equipment, and transmits it to the lower level. Finally, by applying continuous optimization iterations, the optimal scheduling strategy of BIES and the optimal charging and discharging state of the battery under the optimal economic conditions are produced.

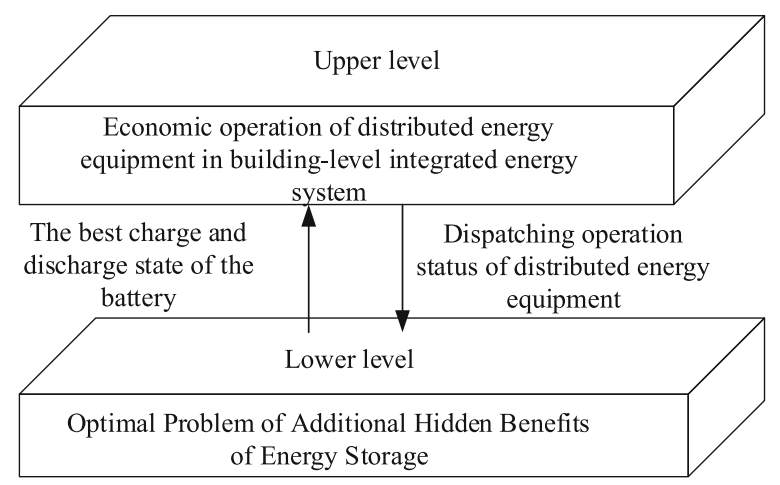

Fig. 2 Transfer relationship of decision variables in the upper and lower levels

\subsection{Upper-level model}

The upper-level model can be represented by:

$$
\begin{aligned}
& \min F_{\mathrm{up}}=F_{\mathrm{fu}}+F_{\mathrm{m}}-F_{\mathrm{ex}} \\
& F_{\mathrm{fu}}=\sum_{t=1}^{T}\left(P_{G T}^{g}(t)+P_{G B}^{g}(t)\right) \rho_{\text {gas }} \\
& F_{\mathrm{m}}=\sum_{t=1}^{T} \sum \alpha_{i} P_{i}(t) \\
& \left.F_{\mathrm{ex}}=\sum_{t=1}^{T}\left(\rho_{\mathrm{se}}(t)+\rho_{\mathrm{be}}(t)\right)-\rho_{\mathrm{be}}(t)\right) \mid \frac{P_{\mathrm{ex}}(t)}{2}+ \\
& P_{\mathrm{ex}}(t)=P_{\mathrm{ES}}^{\mathrm{ch}}(t)-P_{\mathrm{ES}}^{\mathrm{dis}}(t)-P_{\mathrm{NGS}}(t)
\end{aligned}
$$

In the above equations, $\mathrm{T}$ is the total time of the scheduling cycle. $F_{\mathrm{fu}}$ is the cost of natural gas consumption of gas turbines and gas boilers, $F_{\mathrm{m}}$ is the operating cost of each piece of equipment, and $F_{\text {ex }}$ is the interactive benefit between the BIES and power grid. $\alpha_{i}$ and $P_{i}(t)$ are the operating cost of equipment $i$ and the output power at time $t$, respectively. $P_{\mathrm{ex}}(t)$ is the interactive power between the BIES and power grid at time $t$. $P_{\text {ex }}(t)>0$ means that the BIES sends power to the distribution network whereas $P_{\mathrm{ex}}(t)<0$ means that the BIES purchases electricity from the distribution network.

\subsection{Lower-level model}

In order to incorporate the value-added role of batteries in a BIES and formulate a reasonable and effective dispatch strategy, the lower-level model introduces the additional potential income model of electricity storage to optimize the unknown output. Seeking the highest economic benefit brought by the additional potential benefits of electricity storage is equivalent to finding the smallest value of $F_{\text {low. }} . F_{\mathrm{ES}}$ is shown in (12) and there is

$$
\min F_{\text {low }}=\min \left(-F_{\mathrm{ES}}\right)
$$

The lower model can obtain the optimal charge and discharge strategy of the battery, and it is returned to the upper model to continue iterative optimization.

\subsection{Operational constraints}

\subsubsection{Power balance constraint}

$$
\begin{aligned}
P_{\text {buy }}(t) & +\sum_{i} P_{\mathrm{GT} . i}^{\mathrm{e}}(t)+P_{\mathrm{PV}}(t)+P_{\mathrm{WT}}(t)+P_{\mathrm{ES}}^{\mathrm{dis}}(t) \\
= & P_{\text {sell }}(t)+P_{\mathrm{EC}}^{\mathrm{e}}(t)+P_{\mathrm{ES}}^{\mathrm{ch}}(t)+P_{\mathrm{load}}^{e}(t)
\end{aligned}
$$

$$
\sum_{i} P_{\mathrm{GT} . i}^{\mathrm{h}}(t)+\sum_{i} P_{\mathrm{GB} . i}^{\mathrm{h}}(t)=P_{\mathrm{AC} . i}^{\mathrm{h}}(t)+P_{\mathrm{load}}^{\mathrm{h}}(t)
$$




$$
P_{\mathrm{EC}}^{\mathrm{c}}(t)+P_{\mathrm{AC}}^{\mathrm{c}}(t)=P_{\text {load }}^{\mathrm{c}}(t)
$$

where $P_{\text {buy }}(t)$ and $P_{\text {sell }}(t)$ are the electric power purchased and sold by the BIES from the power grid at time $t$, respectively. $P_{\text {load }}^{\mathrm{h}}(t)$ and $P_{\text {load }}^{\mathrm{c}}(t)$ are the respective heating load and cooling load of the BIES at time $t$.

\subsubsection{Equipment upper and lower limits}

$$
P_{i_{-} \min }<P_{i}<P_{i_{-} \max }
$$

where $P_{i_{-} \max }$ and $P_{i_{-} \min }$ are the upper and lower limits of the output of distributed energy equipment $i$, respectively.

\subsubsection{Interactive power constraint}

$$
P_{\text {ex }_{-} \text {min }} \leq P_{\mathrm{ex}} \leq P_{\mathrm{ex}_{-} \max }
$$

where $P_{\text {ex } \_ \text {max }}$ and $P_{\text {ex } \_ \text {min }}$ are the upper and lower limits of the interactive power between the BIES and the power grid, respectively. These are related to the overload capacity of the tie line.

\subsubsection{Battery constraints}

$$
\begin{aligned}
& P_{\mathrm{ES}_{-} \text {min }}^{\mathrm{dis}} \leq P_{\mathrm{ES}}^{\mathrm{dis}}(t) \leq P_{\mathrm{ES}_{-} \text {max }}^{\mathrm{dis}} \\
& P_{\mathrm{ES}_{-} \text {min }}^{\mathrm{ch}} \leq P_{\mathrm{ES}}^{\mathrm{ch}}(t) \leq P_{\mathrm{ES}_{-} \text {max }}^{\mathrm{ch}} \\
& S_{E S}^{\min } \leq S_{E S}(t) \leq S_{E S}^{\max } \\
& S_{\mathrm{ES}}^{\min }-S_{\mathrm{ES}}^{0} \leq \sum_{t=1}^{T} \eta_{\mathrm{ES}}^{\mathrm{ch}} P_{\mathrm{ES}}^{\mathrm{ch}}(t)-\frac{P_{\mathrm{ES}}^{\mathrm{dis}}(t)}{\eta_{\mathrm{ES}}^{\mathrm{dis}}} \leq S_{\mathrm{ES}}^{\max }-S_{\mathrm{ES}}^{0} \\
& S_{\mathrm{ES}}^{0}=S_{\mathrm{ES}}(T)\left(1-\sigma_{\mathrm{ES}}^{\text {loss }}\right)+ \\
& \left(\eta_{\mathrm{ES}}^{\mathrm{ch}} P_{\mathrm{ES}}^{\mathrm{ch}}(T)-\frac{P_{\mathrm{ES}}^{\text {dis }}(T) I_{\mathrm{ES}}^{\mathrm{dis}}(T)}{\eta_{\mathrm{ES}}^{\mathrm{dis}}}\right)
\end{aligned}
$$

where $P_{\mathrm{ES} \text { max }}^{\text {dis }}$ and $P_{\mathrm{ES} \text { min }}^{\text {dis }}$ are the upper and lower limits of battery discharge power, respectively. $P_{\mathrm{ES}_{-} \text {max }}^{\text {ch }}$ and $P_{\mathrm{ES}-\text { min }}^{\text {ch }}$ are the upper and lower limits of battery charging power, respectively. $S_{\mathrm{ES}}^{\max }$ and $S_{\mathrm{ES}}^{\min }$ are the upper and lower limits of the battery capacity, respectively. $S_{\mathrm{ES}}^{0}$ is the initial capacity of the battery, and $S_{\mathrm{ES}}(T)$ is the remaining power in the battery at time $\mathrm{T}$, where $\mathrm{T}=24$. The capacity of the battery is the same at the beginning and end of the dispatch period, and this constraint makes it convenient for the dispatch in the next period.

\subsection{Solving steps}

The above model is a mixed integer nonlinear optimization problem. This paper uses a particle swarm optimization algorithm (PSO) to solve the bi-level optimal operation problem. The model establishment and solution process can be summarized as follows.
Step 1: Input the operating parameters of the energy production, energy conversion and energy storage equipment of the BIES, system maintenance cost, various cost and user load parameters;

Step 2: Set the basic parameters of PSO, such as number of iterations, number of particles, learning factor and inertia weight, etc.

Step 3: Solving the upper-level problem of the model.

(1) Use gas turbine output as the decision variable and set operating constraints for each distributed device. Initialize the population $\mathrm{P}_{0}$ and determine the output power of other distributed equipment according to the load demand and the output of renewable energy during the corresponding period. Calculate the optimal economic cost of the BIES as the fitness value.

(2) Compare the individual extremum $p_{-}$best and the fitness value, the global extremum $g_{-}$best and the fitness value of each particle, and update the current global extreme value and the global optimal position.

(3) The speed and position of the particles are updated by (31)-(33). This paper adopts a linear decrement to adjust the weight so as to improve the optimization efficiency and accelerate the convergence speed.

$$
\begin{aligned}
& v_{i d}(t+1)=w v_{i d}(t)+\eta_{1} \operatorname{rand}()\left[p_{\text {b best }}-z_{i d}(t)\right] \\
& +\eta_{2} \operatorname{rand}()\left[g_{\text {_best }}-z_{i d}(t)\right] \\
& z_{i d}(t+1)=z_{i d}(t)+v_{i d}(t)
\end{aligned}
$$

where $\eta_{1}$ and $\eta_{2}$ are learning factors, and both are taken as $2 . v_{i d}$ is the particle velocity, $z_{i d}$ is the particle position and $w$ is the inertia weight.

$$
w_{t}=w_{\max }-t \times\left(w_{\max }-w_{\min }\right) / N
$$

where $w_{\max }$ and $w_{\min }$ are the maximum and minimum values of inertia weight, respectively. $N$ is the number of iterations.

Step 4: Repeat Step 3. After meeting the error standard, the dispatching strategy of distributed energy equipment is generated and transmitted to the lower level.

Step 5: Solving the lower-level problem of the model.

(1) Use battery output as a decision variable. Initialize the population $P_{0}^{\prime}$ and calculate the optimal additional potential income of electricity storage as the fitness value.

(2) Same as Step 3 (2) (3). 


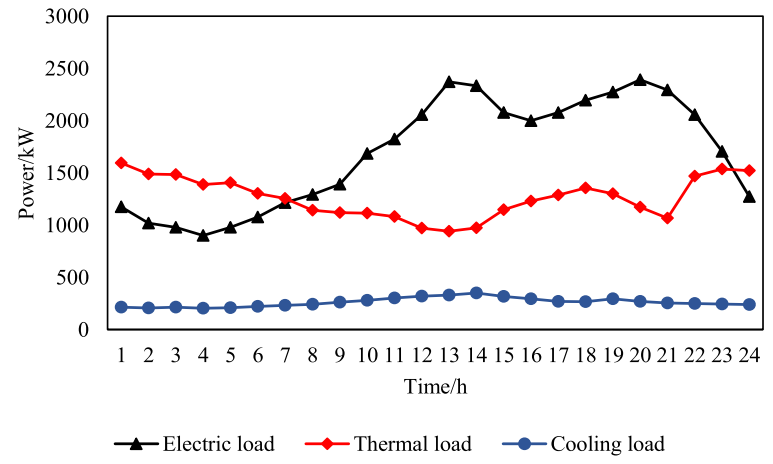

Fig. 3 Typical daily load demand in winter

Step 6: Repeat Step 3. After meeting the error standard, the optimal charge and discharge strategy of the battery is generated considering the system load shortage and real-time electricity price, and transmitted to the upper level to continue the iterative solution.

Step 7: Output the global optimal solution set.

\section{Case analysis}

In order to verify the feasibility and superiority of the proposed optimization model, a typical winter day in a certain area in the north is selected as the research scenario. Cold, heat and electricity demand are considered simultaneously. Taking $\mathrm{T}=24 \mathrm{~h}$ and $\Delta t=1 \mathrm{~h}$ as example, the load demand on a typical day in this area is shown in Fig. 3.

The time-of-use electricity price of energy under gridconnected conditions is shown in Table 1. The economic and technical parameters of equipment are shown in Table 2, and the relevant parameters of energy storage equipment are shown in Table 3.

Assuming that the real-time electricity price remains unchanged within one hour, the following four operating strategies are adopted.

Strategy 1: The battery does not run, and the renewable energy, gas turbine and interactive power meet the generation shortage in the microgrid. Strategy 2: When the battery is running, the charging or discharging state is determined by the generation

Table 1 Energy time-of-use price in the dispatch period

\begin{tabular}{|c|c|c|c|c|}
\hline \multirow[t]{2}{*}{ Type } & \multirow[t]{2}{*}{ Unit } & \multirow{2}{*}{$\begin{array}{l}\text { Peak } \\
\text { 08:00-11:00 } \\
\text { 17:00-22:00 }\end{array}$} & Normal & Valley \\
\hline & & & $\begin{array}{l}07: 00-08: 00 \\
\text { 11:00-17:00 } \\
22: 00-23: 00\end{array}$ & $23: 00-7: 00$ \\
\hline Purchase electricity & $¥ /(k W \cdot h)$ & 0.99 & 0.53 & 0.17 \\
\hline Selling electricity & $¥ /(k W \cdot h)$ & 0.72 & 0.41 & 0.12 \\
\hline Natural gas & $¥ / \mathrm{m}^{3}$ & 2.9 & 2.9 & 2.9 \\
\hline
\end{tabular}

Table 2 Economic and technical parameters of each equipment

\begin{tabular}{llllll}
\hline Equipment & $\boldsymbol{P}_{\boldsymbol{i}_{\mathbf{m} \text { min }} / \mathbf{k W}}$ & $\boldsymbol{P}_{\boldsymbol{i}_{\mathbf{m a x}}} / \mathbf{k W}$ & $\boldsymbol{\eta}^{\mathbf{e}}$ & $\boldsymbol{\eta}^{\boldsymbol{h}} / \boldsymbol{\eta}^{\boldsymbol{c}}$ & $\boldsymbol{a}_{\boldsymbol{i}} /(¥ \mathbf{F} \mathbf{k W})$ \\
\hline PV & 0 & 1500 & - & - & 0.0235 \\
WT & 0 & 1500 & - & - & 0.0196 \\
GT & 120 & 2000 & 0.3 & 0.69 & 0.059 \\
GB & 0 & 2000 & - & 0.9 & 0.026 \\
EC & 0 & 500 & - & 4 & 0.013 \\
AC & 0 & 500 & - & 1.2 & 0.013 \\
\hline
\end{tabular}

shortage instead of the real-time electricity price changes.

Strategy 3: When the battery is running, the charging or discharging state is determined by the real-time electricity price changes instead of the generation shortage. Strategy 4: When the battery is running, the charging or discharging state is determined by both the internal generation shortage and the real-time electricity price changes.

MATLAB programming is used to implement the system operation strategy under different operation scheduling modes during the day, as shown in Fig. 4, including new energy output, gas turbine output, interactive power with the distribution network, and electricity demand within the BIES. The input, conversion and interaction of electric energy under different operation scheduling modes are shown in Fig. 5.

From Figs. 4a-d and 5, it can be seen that the scheduling plan in the optimal scheduling period can meet the electrical load demand and heat load demand of the BIES. It can be seen from the comparison of the four operation scheduling strategies that 00:00-05:00 is the valley period of time-of-use electricity price. The dispatch results in Fig. 4 show that wind power output is relatively high. In order to minimize the system's generation of power being fed back to the power grid during the electricity price valley period, the battery is in a charging state during this stage. When the wind power output does not meet users' power demand, the more economical purchase of electricity from the distribution network is considered to meet the power load demand.

It can be observed from Figs. 4 and 5 that Strategies 2, 3 , and 4 all contribute to peak shaving and valley filling. In Strategy 2, the battery focuses on meeting the BIES load demand. Therefore, during the periods 12:00-15:00 and 19:00-21:00 with a large generation shortage, the battery is in a discharge state, while during the period

Table 3 Related parameters of energy storage equipment

\begin{tabular}{lcllc}
\hline$\sigma_{E S}^{\text {loss }}$ & $\eta_{E S}^{\text {ch }} / \eta_{E S}^{\text {dis }}$ & $P_{E S_{-} \text {max }}^{\text {ch }}(t) / k W$ & $P_{E S_{-} \text {max }}^{\text {dis }}(t) / k W$ & $\boldsymbol{a}_{i} /(¥ / \mathbf{k W})$ \\
\hline 0.01 & 0.95 & 200 & 400 & 0.013 \\
\hline
\end{tabular}




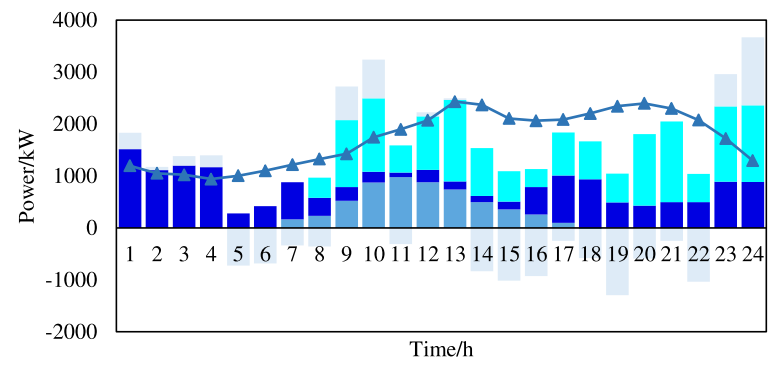

(a) Strategy 1

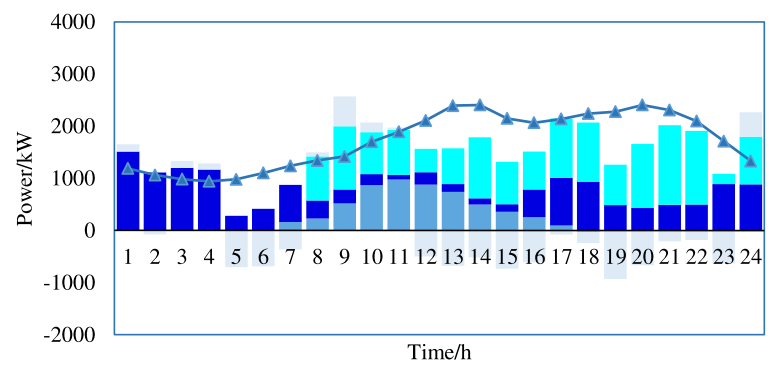

(b) Strategy 2

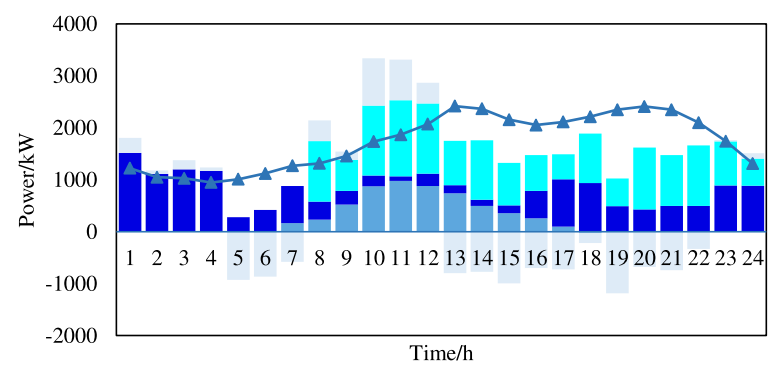

(c) Strategy 3

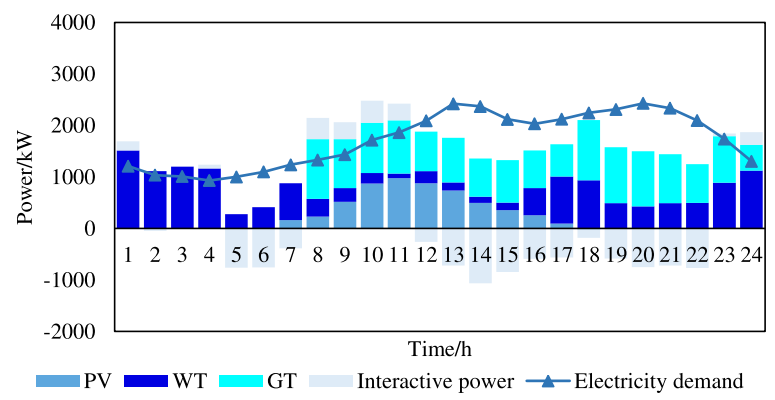

(d) Strategy 4

Fig. 4 Optimization operation results of BIES under different operation scheduling modes during a day

00:00-04:00 when the generation shortage is low, the battery is charged, thereby saving electricity purchase costs. In Strategy 3, the battery focuses on adjusting charge and discharge following the changes in real-time electricity prices. Compared with Strategy 2, Strategy 3 does not consider the generation shortage. During the valley electricity price period 00:00-07:00 and the normal electricity price period 13:00-17:00, the battery is charged to reduce the cost of the BIES. During the peak electricity price period 09:00-11:00 and 18:00-22:00, the battery is discharged to obtain more additional benefits. In Strategy 3, due to the incentive of the time-of-use electricity price, the storage battery responds to the change of the real-time electricity price and successfully transfers electric energy from the valley period to the peak period. In Strategy 4, the storage battery responds to the real-time electricity price with the consideration of the load demand change, so the scheduling method is between Strategies 2 and 3.

The correlation coefficient of the real-time electricity price and generation shortage is shown in Fig. 6. The 18: 00-22:00 period has a high discharge coefficient during this period, the generation shortage is large and the realtime electricity price is at a peak, and hence, the battery is discharged. In contrast, the period 00:00-04:00 has the largest charging coefficient. In Strategy 4, the battery charging and discharging decision corresponds to the correlation coefficient, and the battery is charged during the period of low electricity price from 00:00-07:00. However, in Strategy 3, the battery is charged between 04:00-07:00, that is closer to the peak electricity price period of discharge which can reduce the loss of electric energy. In Strategy 4, the battery is mainly charged at 00:00-04:00, as because of the high wind power output and low generation shortage during this period, charging is beneficial for realizing the energy transport function of the battery and smoothing the load fluctuation of the BIES. During the peak electricity price period from 09:00 to 11:00, there are also differences between Strategies 3 and 4 in battery scheduling strategies. In Strategy 3, the battery discharges more electricity during the period from 09:00 to 10:00, when the electricity price is higher, which reduces the loss of electric energy. In Strategy 4, it considers the impact of the generation shortage and focuses on the discharge at 10:00-11:00, which is more conducive to the stability of BIES.

During the normal-price period 13:00-17:00, the battery charging intensity in Strategy 4 is much lower than in Strategy 3, as the generation shortage during this period is relatively large. This is because Strategy 3 only considers the advantages of real-time electricity prices for charging, which is not conducive to the stability of the BIES. Through the comparative analysis, it can be seen that the additional potential revenue model of electricity storage can fully use the electricity price difference to reduce the operating cost while meeting the electricity demand of users. Thus, the potential of battery participation in market services is tapped. 


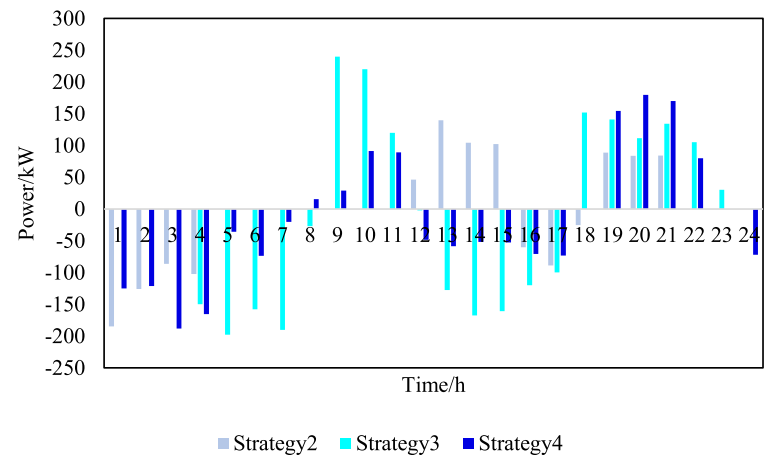

Fig. 5 Battery output results under different operation scheduling modes during a day

Table 4 shows the scheduling results under the four different scheduling strategies. It can be seen from the total cost that the proposed operating mode that considers the additional potential benefits of electricity storage has the lowest economic cost. The proposed scheduling Strategy 4 saves $7 \%$ over Strategy 1, 3\% over Strategy 2, and $2 \%$ over Strategy 3. It demonstrates that using a reasonable and effective strategy to plan battery charging and discharging can effectively exploit the potential of energy storage, reduce the total cost of the system and improve the economic benefits.

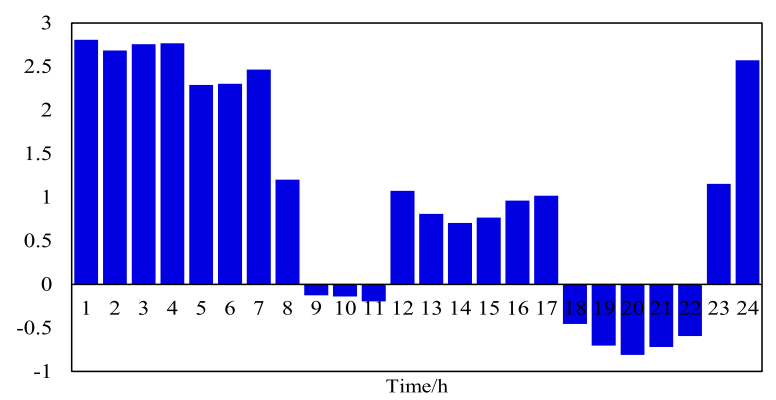

(a) Discharge correlation coefficient $\mathrm{A}_{\mathrm{NGS}}$

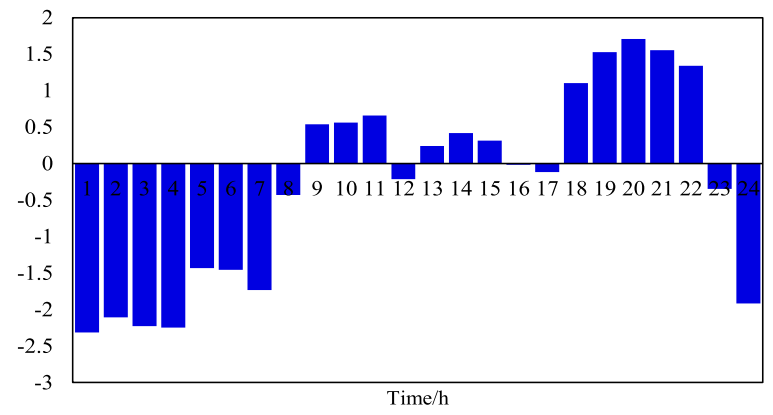

(b) Charging correlation coefficient $\mathrm{B}_{\mathrm{NGS}}$

Fig. 6 The correlation coefficient between battery charge and discharge, generation shortage and real-time electricity price in Strategy 4
Table 4 Scheduling results under different operation scheduling modes

\begin{tabular}{lllll}
\hline Cost/\# & Strategy $\mathbf{1}$ & Strategy $\mathbf{2}$ & Strategy $\mathbf{3}$ & Strategy $\mathbf{4}$ \\
\hline$F_{\text {fu }}$ & $16,549.99$ & $15,819.13$ & $15,774.40$ & $15,349.10$ \\
$F_{\mathrm{m}}$ & 1252.59 & 1137.37 & 1157.71 & 1056.56 \\
$F_{\text {ex_sell }}$ & 1626.61 & 711.57 & 1657.78 & 1230.99 \\
$F_{\text {ex_buy }}$ & 5980.25 & 4960.71 & 5623.98 & 5320.79 \\
$F$ & $22,156.22$ & $21,205.64$ & $20,898.31$ & $20,495.46$ \\
\hline
\end{tabular}

\section{Conclusion}

In this paper, the form and model of additional potential income from energy storage are analyzed in the context of a BIES. Combining the physical structure and energy consumption characteristics of a BIES, a bi-level optimal operation model of a BIES considering the additional potential benefits of energy storage is established. Aiming at optimal economic efficiency, the economic operation of the battery under different dispatching modes is analyzed. The results show that the proposed scheduling strategy saves $7 \%$ over Strategy 1, 3\% over Strategy 2, and 2\% over Strategy 3 in cost. The proposed optimization model allows promote energy storage for more active participation in market scheduling. It also helps to optimize the output of each component, and to further improve economic benefit.

\section{Abbreviations}

BIES: Building-level integrated energy system; IES: Integrated energy system; PV: Photovoltaic unit; WT: Wind turbines; GT: Gas turbines; GB: Gas boilers; EC: Electric chiller; AC: Absorption chiller; ES: Electric energy storage;

HRB: Heat recovery boiler

\section{Acknowledgements}

Not applicable.

\section{Authors' contributions}

Sai Liu and Cheng Zhou drafted the manuscript. Yu Liu and Sai Liu provided the idea and other technical guidance required for completing the study. Sai Liu, Cheng Zhou and Haomin Guo participated in the simulation model building. Cheng Zhou, Qingxin Shi and lan Schomer analyzed and interpreted the simulation results. Yu Liu, Qingxin Shi and Tiancheng E. Song reviewed and edited the manuscript. All authors read and approved the final manuscript.

\section{Authors' information}

S. Liu(1993-), male, received the B.S. and M.S. degrees from Southeast University, major in the optimization and operation of integrated energy system and maintenance of substation equipment.

Y. Liu(1990-), male, received the B.S., M.S., and Ph.D. degrees from Southeast University, he was a visiting scholar with the University of Alberta, he is now a lecturer with School of Electrical Engineering, Southeast University, major in power system planning and operation, integrated energy systems, and non-intrusive load monitoring.

Funding

Project Supported by National Nature Science Foundation of China under Grant 51907024 


\section{Availability of data and materials}

The datasets used during the current study are available from the corresponding author on reasonable request.

\section{Competing interests}

The authors declare that they have no competing interests.

\begin{abstract}
Author details
${ }^{1}$ Maintenance Branch Company, State Grid Jiangsu Electric Power Co., Ltd., Nanjing 211102, Jiangsu Province, China. ${ }^{2}$ Kunshan Power Supply Branch Company, State Grid Jiangsu Electric Power Co., Ltd., Kunshan 215300, Jiangsu Province, China. ${ }^{3}$ Xiamen Power Supply Company, State Grid Fujian Electric Power Co., Ltd., Xiamen 361004, Fujian Province, China. ${ }^{4}$ Enliten Lab, University of Tennessee, Knoxville, TN 37996, USA. ${ }^{5}$ School of Electrical Engineering, Southeast University, Nanjing 210096, Jiangsu Province, China.
\end{abstract}

Received: 28 August 2020 Accepted: 28 January 2021

Published online: 23 February 2021

\section{References}

1. Keirstead, J., Jennings, M., \& Sivakumar, A. (2012). A review of urban energy system models: Approaches, challenges and opportunities. Renewable \& Sustainable Energy Review, 16(6), 3847-3866.

2. International Energy Agency. (2012). Energy technology perspectives 2012: pathways to a clean energy system.

3. U.S. Energy Information Administration. (2016). 2016 International Energy Outlook.

4. Huang, Z., Fang, B. L., \& Deng, J. (2020). Multi-objective optimization strategy for distribution network considering $\mathrm{V} 2 \mathrm{G}$-enabled electric vehicles in building integrated energy system. Protection and Control of Modern Power Systems, 5(01), 7.

5. China Building Energy Efficiency Development Report (2016). Building energy efficiency operation management. Beijing: China Construction Industry Press.

6. Chen, H., Li, F. X., \& Wang, Y. R. (2018). Wind power forecasting based on outlier smooth transition autoregressive GARCH model. Journal of Modern Power Systems and Clean Energy, 6(03), 532-539.

7. Gao, S., Liu, S., Liu, Y., Zhao, X., \& Song, T. C. E. (2019). Flexible and economic dispatching of AC/DC distribution networks considering uncertainty of wind power. IEEE Access, 7, 100051-100065.

8. Murty, V. V. S. N., \& Kumar, A. (2020). Multi-objective energy management in microgrids with hybrid energy sources and battery energy storage systems. Protection and Control of Modern Power Systems, 5(01), 1-20.

9. Wang, H., Zhao, J., \& AN, Q.S., \& Kang, L. G. (2015). Study on optimization and policy incentives of distributed energy system under different building loads. Proceedings of the CSEE, 35(14), 3734-3740.

10. Liu, C. H., Zheng, Y., Cai, X., \& Chen, H. (2014). Intelligent economic scheduling for multitype cooling system in high buildings. Modern Building Electric, 5(08), 5-10.

11. Ren, W. S., Gao, H. J., Liu, Y. B., Liu, J. Y., Li, C. Y., \& Liu, X. L. (2019). Optimal day-ahead electricity scheduling and sharing for smart building cluster Power System Technology, 43(07), 2568-2575.

12. Wu, J. C., Ai, X., Zhang, Y., Wang, H. Y., \& Hu, J. J. (2018). Day-ahead optimal scheduling for high penetration of distributed energy resources in community under separated distribution and retail operational environment. Power System Technology, 42(6), 1709-1717.

13. Xu, Q. S., Li, L., Cai, J. L., Luan, K. N., \& Yang, B. (2018). Day-ahead optimized economic dispatch of CCHP multi-microgrid system considering power interaction among microgrids. Automation of Electric Power Systems, 42(21), 36-44.

14. Liu, N., Wang, J., \& Wang, L. F. (2019). Hybrid energy sharing for multiple microgrids in an integrated heat-electricity energy system. IEEE Transactions on Sustainable Energy, 10(03), 1139-1151.

15. Zhang, Y., Zhang, T., Liu, Y. J., \& Guo, B. (2015). Optimal energy management of a residential local energy network based on model predictive control. Proceedings of the CSEE, 35(14), 3656-3666.

16. Fitzpatrick, P., D'Ettorre, F., De Rosa, M., Yadack, M., Eicker, U., \& Finn, D. P. (2020). Influence of electricity prices on energy flexibility of integrated hybrid heat pump and thermal storage systems in a residential building. Energy and Buildings, (223), 1-15.
17. Zhou, C., Zheng, J. Y., Liu, S., Liu, Y., Mei, F., Pan, Y., ... Wu, J. Z. (2019) Operation optimization of Multi-District integrated energy system considering flexible demand response of electric and thermal loads. Energies, 12(20), 3831.

18. Kim, Y. J. (2020). A supervised-learning-based strategy for optimal demand response of an HVAC system in a multi-zone office building. IEEE Transactions on Smart Grid, 11(5), 4212-4226.

19. Junker, R. G., Azar, A. G., Lopes, R. A., Lindberg, K. B., Reynders, G., Relan, R., \& Madsen, H. (2018). Characterizing the energy flexibility of buildings and districts. Appl Energy, 225, 175-182.

20. Wu, X., Wang, X. L., Bie, C. H., \& Wand, J. X. (2013). Economic operation of microgrid with combined heat and power system. Electric Power Automation Equipment, 33(08), 1-6.

21. Wu, X., Wang, X. L., Wand, J. X., \& Bie, C. H. (2013). Economic generation scheduling of a microgrid using mixed integer programming. Proceedings of the CSEE, 33(28), 2-8

22. Li, P., Xu, W. N., Zhou, Z. Y., \& Li, R. (2014). Optimal operation of microgrid based on improved gravitational search algorithm. Proceedings of the CSEE 34(19), 3073-3079.

23. Wang, Z. M., Gu, C. H., Li, F. R., Bale, P., \& Sun, H. B. (2013). Active demand response using shared energy storage for household energy management. IEEE Transactions on Smart Grid, 4(4), 1888-1897.

24. Huang, L. B., Walrand, J., \& Ramchandran, K. (2012). Optimal demand response with energy storage management. In IEEE third international conference on smart grid communications, Tainan, (pp. 61-66).

\section{Submit your manuscript to a SpringerOpen ${ }^{\circ}$ journal and benefit from:}

- Convenient online submission

- Rigorous peer review

- Open access: articles freely available online

- High visibility within the field

- Retaining the copyright to your article

Submit your next manuscript at $>$ springeropen.com 